ISSN 1112-9867

Available online at

http://www.jfas.info

\title{
THE EFFECT OF HIGH TEMPERATURES ON CONCRETE INCORPORATING ULTRAFINE SILICA AND POLYPROPYLENE FIBERS
}

\author{
M. Benkaddour ${ }^{1}$, Y. Senhadji ${ }^{2, *}$, F. Kazi Aoual ${ }^{3}$ and A. Semcha ${ }^{3}$ \\ ${ }^{1}$ Laboratoire Matériaux et Génie Civil, Université Saàd Dahleb, Blida 1, Algérie \\ ${ }^{2}$ Département de Génie Civil, Université de Mascara, Mascara 29000, Algérie \\ ${ }^{3}$ Département de Génie Civil, Laboratoire Lab-Mat, ENP-Oran El Mnaouer 31000, Algérie
}

Received: 04 December 2015 / Accepted: 28 Mars 2016 / Published online: 01 May 2016

\begin{abstract}
In recent years, lots of studies have attempted to examine the possible causes for the thermal instability of ordinary concrete and high performance. However, we still do not know the exact terms of phenomena taking place during exposure to high temperature and the technological solutions that exist (polypropylene fibres, thermal reported) are not always well controlled.

In this work, several concrete formulations have been tested and multi-scale observation of high-temperature behavior of ordinary concrete (compressive strength of $48 \mathrm{MPa}$ ) and HPC (compressive strength $75 \mathrm{MPa}$ ) were adopted.

On the scale of the material, the identification of trends with temperature data such as porosity and particularly the mechanical properties allow us to better understand the behaviour of concrete at high temperature differential thermal analysis have been also made.
\end{abstract}

Keywords: concrete; high temperature; porosity; compressive strength; ultrafine Silica.

Author Correspondence, e-mail: senhadjidz@yahoo.fr

doi: http://dx.doi.org/10.4314/jfas.v8i2.6 


\section{INTRODUCTION}

Dans une approche de prévention des risques des constructions, les structures en béton doivent être conçues de façon à être durablement résistantes et en particulier à résister à une éventuelle élévation de température. Durant ces dernières années de nombreuses recherches ont été entreprises pour comprendre le comportement du béton à haute température. Cette étude expérimentale contribue à ces recherches.

Ce travail consiste à élaborer plusieurs formulations de bétons et à les tester. Plusieurs échelles d'observation du comportement à haute température d'un béton ordinaire (résistance à la compression de $40 \mathrm{MPa})$ et un $\mathrm{BHP}(\mathrm{Rc}$ de $70 \mathrm{MPa})$ ont été adoptées. A l'échelle du matériau, la détermination de l'évolution des caractéristiques avec la température telles que la porosité et particulièrement la résistance à la compression nous permettent de mieux comprendre le comportement du béton soumis aux hautes températures. L'étude a montré une amélioration des propriétés mécaniques et de la durabilité (résistance au feu).

\section{PROGRAMME EXPERIMENTAL}

\subsection{Matériaux utilisés:}

Le ciment utilisé est un CEM II/A 42,5 MPa de la cimenterie LAFARGE de Oggaz (Ouest Algérien). Ses caractéristiques chimiques, minéralogiques et physiques sont présentées dans les tableaux 1, 2 et 3 respectivement.

Tableau 1. Analyse chimique du ciment

\begin{tabular}{lcccccccc}
\hline Les composants & $\mathrm{CaO}$ & $\mathrm{SiO}_{2}$ & $\mathrm{Al}_{2} \mathrm{O}_{3}$ & $\mathrm{Fe}_{2} \mathrm{O}_{3}$ & $\mathrm{MgO}$ & $\mathrm{SO}_{3}$ & $\begin{array}{c}\text { Perte au } \\
\text { feu }\end{array}$ & Total \\
\hline Les teneurs $(\%)$ & 64,13 & 20,39 & 5,96 & 3,57 & 1,13 & 2,57 & 2,37 & 100,12 \\
\hline
\end{tabular}

Tableau 2. Composition minéralogique du clinker selon Bogue

\begin{tabular}{ccccccc}
\hline Phase & \multicolumn{7}{c}{ Clinker } & Régulateur de prise \\
\hline Minéraux & $\mathrm{C}_{3} \mathrm{~S}$ & $\mathrm{C}_{2} \mathrm{~S}$ & $\mathrm{C}_{3} \mathrm{~A}$ & $\mathrm{C}_{4} \mathrm{AF}$ & $\mathrm{CaO}$ libre & gypse \\
Teneur $\quad(\%)$ & 60 & 12,5 & 9,75 & 11 & 0,35 & 5 \\
\hline
\end{tabular}

Le squelette granulaire est formé de deux fractions granulaires (3/8 et 8/16) de la carrière de Kristel (Oran) et un sable 0/4 composé d'un sable concassé, corrigé avec un sable de mer (Terga). Leurs caractéristiques chimico- physiques sont regroupées dans les tableaux 4, 5 et 6. 
Tableau 3. Caractéristiques physiques du ciment

\begin{tabular}{llcl}
\hline \multicolumn{1}{c}{ Caractéristiques physiques } & \multicolumn{1}{l}{ Normes suivies } \\
\hline Ciment anhydre & Mv absolue $\left(\mathrm{g} / \mathrm{cm}^{3}\right)$ & 3,04 & NF P 18 555 \\
& Mv apparente $\left(\mathrm{g} / \mathrm{cm}^{3}\right)$ & 1,76 & NF P 18 555 \\
& Surface spécifique Blaine $\left(\mathrm{cm}^{2} / \mathrm{g}\right)$ & 3200 & NF P 15 442 \\
\cline { 2 - 4 } & Consistance normalisée $(\%)$ & 26 & NA 230 et NF EN 196 \\
\multirow{3}{*}{ Pâte de ciment } & Consistance; C + addition & 24.5 & NA 230 et NF EN 196 \\
& Compacité $(\%)$ & 56.33 & NF EN 196 \\
& Début de prise & $2 \mathrm{~h} \mathrm{40mn}$ & NF 15-301 \\
& Fin de prise & $4 \mathrm{~h}$ & NF 15-301 \\
\hline
\end{tabular}

Un adjuvant plastifiant non coloré réducteur d'eau Viscocrete 3045 (Sika) a été choisi, il est conforme à la norme NF EN 934-2. Ses données techniques sont: le PH égal à $5 \pm 1$, la densité égale à $1,11 \pm 0,02$ et l'extrait sec 36,4 $\pm 1,8 \%$.

Le SIKACRETE HD est l'addition minérale utilisée, c'est une silice ultra fine pour bétons exposés aux milieux agressifs.

Les fibres de polypropylène utilisées sont des fibres monofilament (figure 1), elles sont livrées sous forme d'amas qui se dispersent au cours du malaxage, leurs principales caractéristiques sont présentées dans le Tableau 7.

Tableau 4. Caractéristiques chimiques des granulats

\begin{tabular}{|c|c|c|c|c|c|c|c|c|c|}
\hline Composants & $\mathrm{SiO}_{2}$ & $\mathrm{Al}_{2} \mathbf{O}_{3}$ & $\mathrm{CaO}$ & $\mathrm{Fe}_{2} \mathrm{O}_{3}$ & MgO & $\mathrm{CaCO}_{3}$ & $\mathrm{CO}_{2}$ & $\mathrm{H}_{2} \mathrm{O}$ & $\begin{array}{c}\text { Perte au } \\
\text { feu }\end{array}$ \\
\hline Sable de mer & 70.15 & 0.01 & 16.21 & 0.41 & 0.14 & 29.09 & 12.8 & 0.58 & 13.8 \\
\hline $\begin{array}{l}\text { Sable de } \\
\text { carrière }\end{array}$ & 19.86 & 0.00 & 43.94 & 0.44 & 0.32 & 79.09 & 34.8 & 0.88 & 35.68 \\
\hline G 3/8 & 19.01 & 0.00 & 44.54 & 0.41 & 0.21 & 36.13 & 35.2 & 0.93 & 80.00 \\
\hline G 8/16 & 13.47 & 0.00 & 47.59 & 0.37 & 0.21 & 38.57 & 37.6 & 0.97 & 85.45 \\
\hline
\end{tabular}


Tableau 5. Caractéristiques physiques des sables

\begin{tabular}{|c|c|c|c|}
\hline \multicolumn{2}{|c|}{ Paramètres physiques } & Sable de mer & Sable de carrière \\
\hline \multicolumn{2}{|c|}{ Mv absolue $\left(\mathrm{g} / \mathrm{cm}^{3}\right)$} & 2.63 & 2.58 \\
\hline Mv apparente & $\left(\mathrm{g} / \mathrm{cm}^{3}\right)$ & 1.67 & 1.62 \\
\hline \multirow{2}{*}{$\begin{array}{l}\text { Equivalent de } \\
\text { sable }\end{array}$} & Visuel & 87.90 & 97.80 \\
\hline & Piston & 83.64 & 92.28 \\
\hline \multicolumn{2}{|c|}{ Module de finesse } & 1.8 & 3 \\
\hline \multicolumn{2}{|l|}{ Compacité (\%) } & 57.57 & 61 \\
\hline
\end{tabular}

Tableau 6. Caractéristiques physiques des graviers

\begin{tabular}{lccc}
\hline Paramètres physiques & Unités & G 3/8 & G 8/16 \\
\hline Mv absolue & $\left(\mathrm{g} / \mathrm{cm}^{3}\right)$ & 2.54 & 2.67 \\
Mv apparente & $\left(\mathrm{g} / \mathrm{cm}^{3}\right)$ & 1.59 & 1.59 \\
Compacité & $(\%)$ & 59 & 52 \\
teneur en eau & $(\%)$ & 0.37 & 1.18 \\
Coef de propreté & $(\%)$ & 6.78 & 11.11 \\
Coef d'absorption & - & 0.98 & 0.86 \\
Coef volumique & - & 0.23 & 0.23 \\
Coef de Los Angeles & - & 22 & 24 \\
Coef Micro Deval & $(\%)$ & 16 & 16 \\
\hline
\end{tabular}


Tableau 7. Caractéristiques des fibres

\begin{tabular}{lc}
\hline \multicolumn{2}{c}{ Fibres de polypropylène monofilament } \\
\hline Diamètre $(\mu \mathrm{m})$ & 18 \\
Longueur $(\mu \mathrm{m})$ & 12 \\
Masse volumique $\left(\mathrm{kg} / \mathrm{m}^{3}\right)$ & 912 \\
Température de fusion $\left({ }^{\circ} \mathrm{C}\right)$ & 171 \\
Température de vaporisation $\left({ }^{\circ} \mathrm{C}\right)$ & 347 \\
\hline
\end{tabular}

\subsection{Méthodes expérimentales}

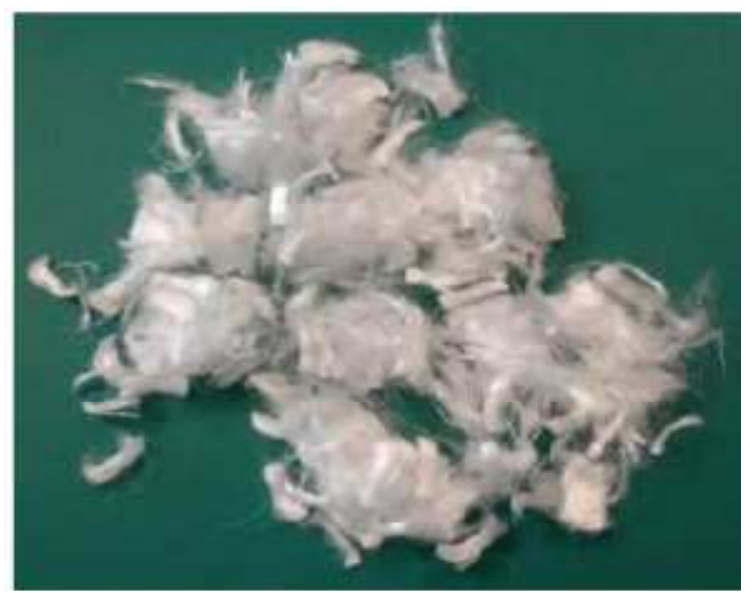

Fig.1. Fibres de polypropylène

\subsubsection{Fabrication et conservation des éprouvettes}

Suite à une longue série d'essais, en jouant sur plusieurs paramètres tels que $C, E / C$ et $G / S$, et suivant la méthode de Dreux et la norme EN 206-1, on a obtenu une bonne formulation d'un béton ordinaire (BO). Suivant la norme NF P 18-358, le dosage en adjuvant a été défini et un BA (béton avec adjuvant) a été fabriqué, ce dernier a été amélioré pour obtenir le BAF (béton avec adjuvant et silice ultra fine), et le BAFP (béton avec adjuvant, silice ultra fine et fibres de polypropylène).

Tableau 8. Désignations et formulations des bétons conféctionnés

\begin{tabular}{lcccc}
\hline Composants & BO & BA & BAF & BAFP \\
\hline Ciment $\left(\mathrm{kg} / \mathrm{m}^{3}\right)$ & 475 & 475 & 475 & 475 \\
Sable 0/4 $\left(\mathrm{kg} / \mathrm{m}^{3}\right)$ & 582 & 604 & 604 & 604 \\
Graviers $3 / 8\left(\mathrm{~kg} / \mathrm{m}^{3}\right)$ & 338 & 352 & 352 & 352 \\
Graviers $8 / 16\left(\mathrm{~kg} / \mathrm{m}^{3}\right)$ & 760 & 792 & 792 & 792 \\
Eau $\left(1 / \mathrm{m}^{3}\right)$ & 213.75 & 190 & 190 & 190 \\
Superplastifiant $(\%$ ciment $)$ & - & 0.5 & 0.5 & 0.5 \\
Silice ultra fine $\left(\mathrm{kg} / \mathrm{m}^{3}\right)$ & - & - & 15 & 15 \\
Polypropylène $\left(\mathrm{kg} / \mathrm{m}^{3}\right)$ & - & - & - & 2 \\
E/C & 0.45 & 0.40 & 0.40 & 0.40 \\
\hline
\end{tabular}

Notre étude a été faite sur des cubes de $10 \times 10 \times 10 \mathrm{~cm}^{3}$. Le coulage a été effectué grâce à un malaxeur électrique (50 litres/gâchée de béton). Le déroulement des gâchées est passé par un pré-mouillage du malaxeur, mélange à sec des constituants solides (granulats, ciment, addition, 
fibres de polypropylène), ajout du mélange de 2/3 d'eau puis 1/3 d'eau plus adjuvant.

Après démoulage, les éprouvettes ont été conservées dans des bassins d'eau saturés en chaux à une température de $20^{\circ} \mathrm{C}\left( \pm 2{ }^{\circ} \mathrm{C}\right)$ environs jusqu'au jour de l'essai.

\subsubsection{Propriétés du béton à l'état durci}

- Le chargement mécanique du béton

Les essais de la résistance à la compression ont été faits suivant les normes NA 5093, EN12 390 et NF P 455 à 7, 28 et 60 jours à l'aide d'une presse qui répond à la norme EN 12390-4, chaque éprouvette a été soumise à une charge croissante jusqu'à la rupture.

\section{- Le chargement thermique puis mécanique du béton}

Pour chauffer les éprouvettes du béton nous avons utilisé un four à moufle d'une capacité de chauffage de $1400^{\circ} \mathrm{C}$.

Lors un incendie, il y a des courants d'air très important, et par l'intervention des sapeurs pompiers, le béton exposé au feu est arrosé de l'eau. Cela nous a poussés de simuler cette opération par deux types de choc thermiques, un choc thermique dans l'air et un choc thermique dans l'eau. Les détails des essais sont montrés par le schéma suivant (figure 2) :

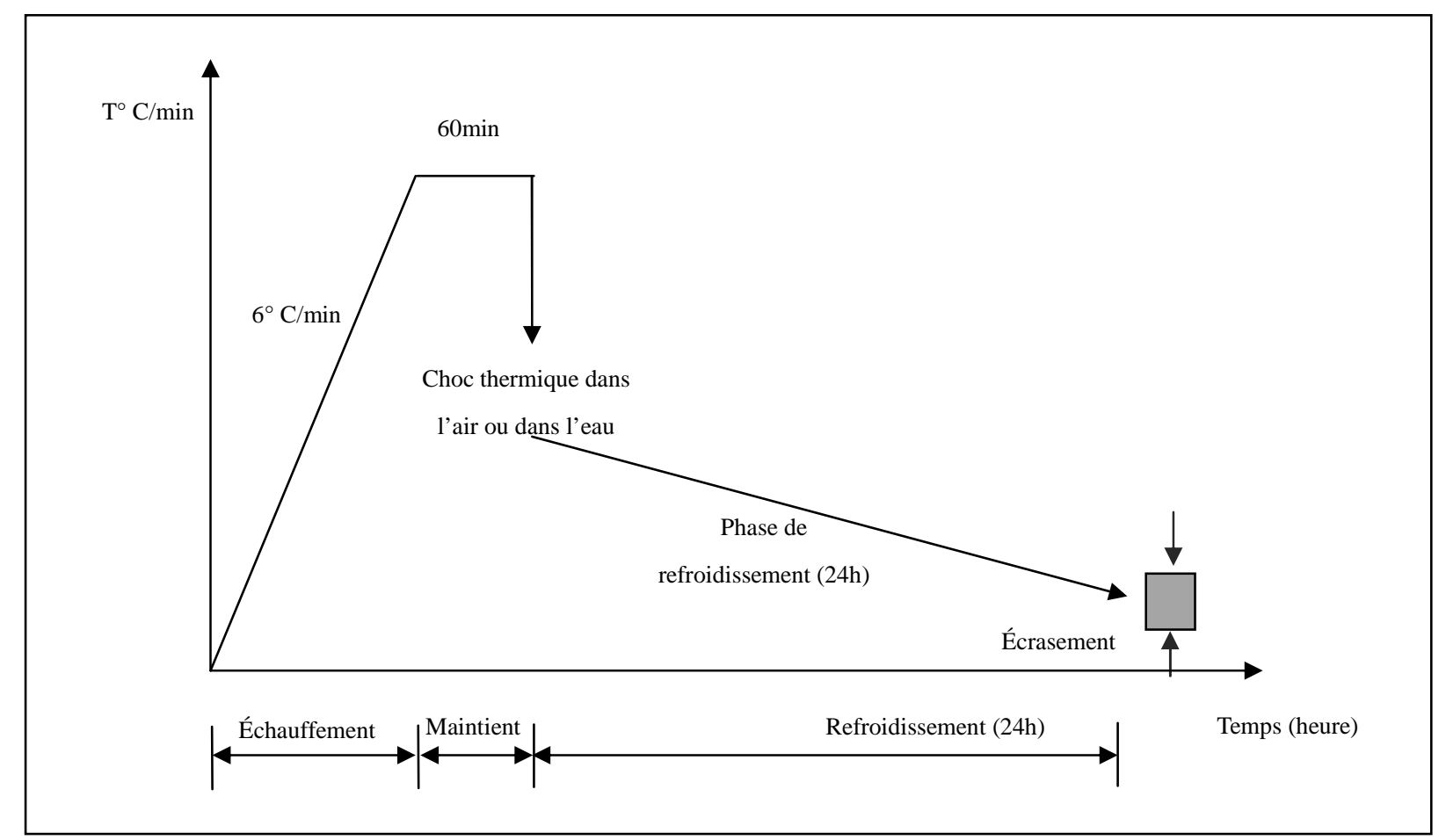

Fig.2. Cycle de chauffage des éprouvettes du béton (Choc thermique dans l'air ou dans l'eau - Refroidissement - Ecrasement) 


\section{- La porosité ouverte du béton (ASTM C642)}

Il s'agit de découper les cubes de $100 \mathrm{~mm}$ d'arrête en 8 petits cubes de $5 \mathrm{~cm}$, les sécher à $103^{\circ} \mathrm{C}$ jusqu'à masse constante (pendant au moins 24 heures), noter la masse (A), les immerger dans une casserole d'eau, les chauffer jusqu'à ébullition, et ce pendant 5 heures. Essuyer et peser l'échantillon, soit une masse (B).

La porosité ouverte à l'eau, exprimée en pourcentage, est définie alors comme :

$$
P(\%)=\frac{(B-A)}{B} \times 100
$$

\section{RESULTATS ET DISCUTIONS}

\subsection{La porosité à température ambiante et après chauffage à $250^{\circ} \mathrm{C}$}

A température ambiante la porosité ouverte des différents bétons varie. Les bétons BA, BAF et BAFP ont une porosité faible par rapport à celle du BO. Pour le BA cette porosité de $5 \%$ de différence est du à la diminution de dosage en eau. Concernant le BAF et le BAFP en plus de leur dosage en eau faible, ils sont fabriqués avec de la silice ultra fine, d'où une porosité de la matrice moins importante que pour le BO et le BA.

En comparant la porosité des bétons sans addition (BO et BA) avec ceux avec addition, on trouve une différence de $40 \%$ par rapport au BAF et de $25 \%$ par rapport au BAFP. La porosité de BAF est plus grande que celle de BAFP de $20 \%$ environs (voir figure 3). Ce dernier contient en supplément $2 \mathrm{~kg}$ de fibre de polypropylène. Le serrage insuffisant du BAFP lors de sa mise en place a favorisé la création d'une porosité de $20 \%$ par rapport au BAF Selon Mindeguia [1]. Aussi l'incompatibilité des fibres avec la pâte liante à la formulation peut crées une certaine porosité [2]. 


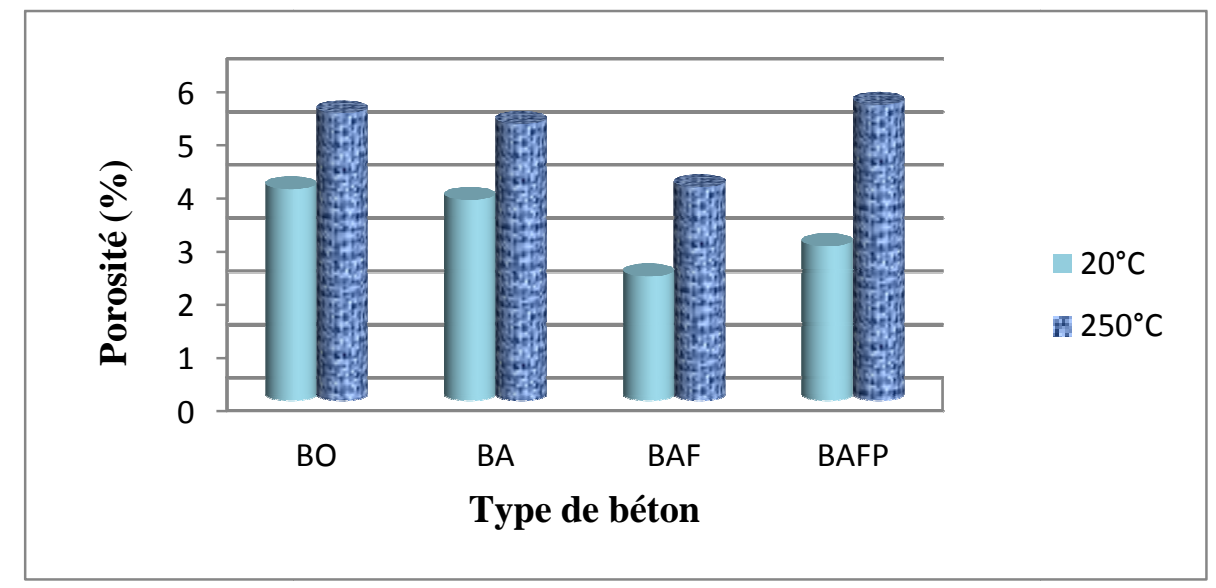

Fig.3. Effet de la température sur la porosité ouverte des bétons étudiés

La porosité du $\mathrm{BAF}$ après chauffages à $250^{\circ} \mathrm{C}$ reste toujours faible par rapport au $\mathrm{BO}$ et $\mathrm{BA}$ mais celle du BAFP augmente significativement de 2.90 à $5.56 \%$, cette amélioration de 52\% environs est due aux fibres de polypropylène. Ces derniers fondent durant le chauffage à une température de $171^{\circ} \mathrm{C}$ environs [3,4]. Des expériences ont montré que les fibres ainsi fondues étaient absorbées par la pâte de ciment durcie [3-5], par conséquence, un vide a été crée à l'endroit initial de la fibre, offrant un réseau poreux à la pâte liante [5].

La fibre n'a pas besoin d'être entièrement fondue pour procurer une porosité supplémentaire, la mauvaise adhésion entre la fibre et la matrice cimentaire cause aussi une porosité [4].

La fonte des fibres s'accompagne également des fissures. Ceci peut s'expliquer par le fait que les fibres se dilatent de $10 \%$ en fondant, ce qui peut générer des contraintes et par la suite une formation des fissures [1,5].

\subsection{Influence du choc thermique sur la porosité}

La porosité des échantillons choqués dans l'air et plus élevée par rapport à ceux qui ont subit un choc dans l'eau pour tous les bétons, surtout dans les cas du BAF, la différence enregistrée est de $44 \%$. Celle de ce dernier a diminuée de $3.8 \%$ par rapport au BAF non chauffé. Concernant les autres mesures de la porosité, celle du BO choqué dans l'eau diminue de $15 \%$, du BA diminue de 5\% tandis de celle du BAFP diminue significativement de $27 \%$ par rapport aux BAFP choqués dans l'air. 


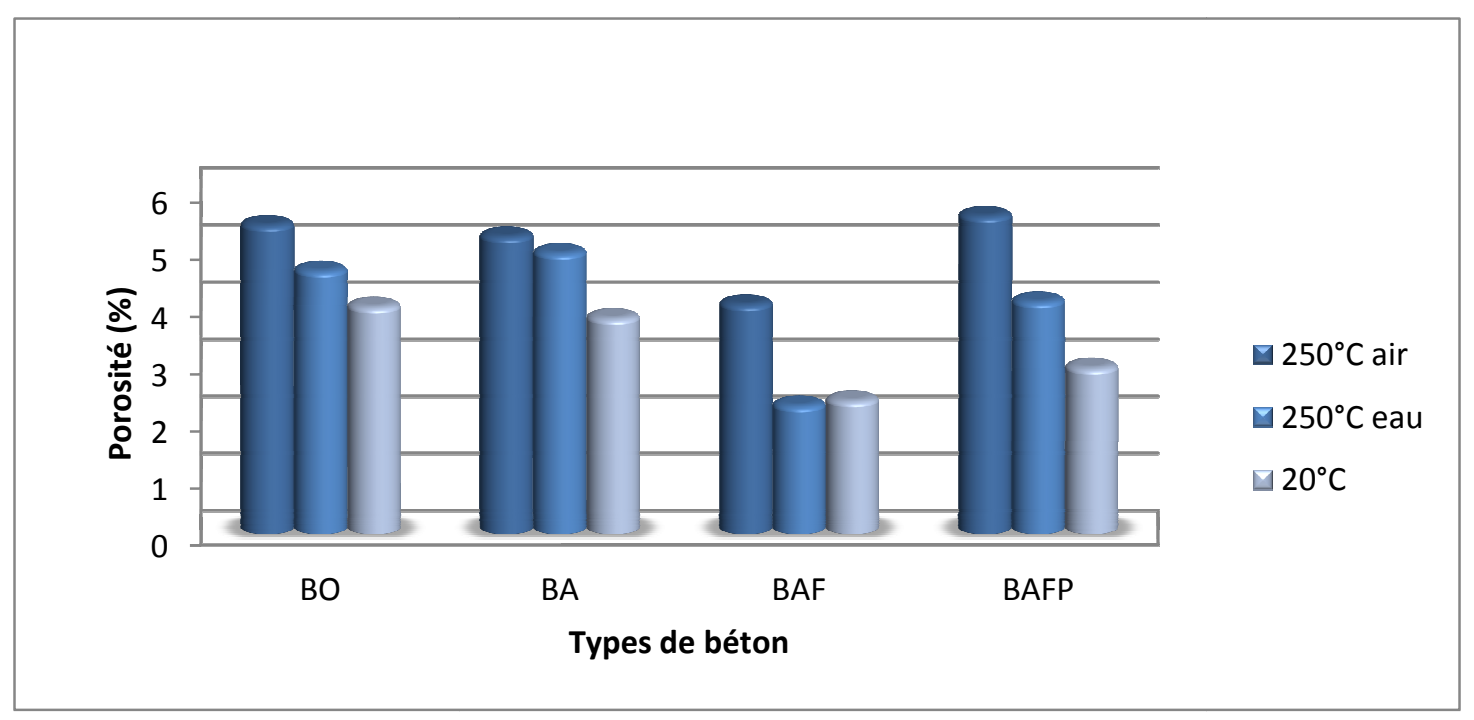

Fig.4. Porosité ouverte des différents bétons étudiés selon la nature du refroidissement

La porosité du béton augmente avec la température, cette croissance est associée au départ d'eau en créant des vides. Lors de l'immersion de ces bétons dans l'eau juste après leur chauffage, ces vides sont remplis d'une quantité d'eau qui varie en fonction de la porosité avant l'introduction dans l'eau et des tailles des pores. Cette eau réagit avec le produit de la déshydratation (la chaux) suivant la réaction suivante :

$$
\mathrm{CaO}+\mathrm{H}_{2} \mathrm{O} \rightarrow \mathrm{Ca}(\mathrm{OH})_{2}
$$

Le produit de cette réaction est la portlandite, qui possède un volume plus important que la $\mathrm{CaO}$. Ce qui diminue la porosité $[1,6]$.

\subsection{Influence de la température sur la résistance à la compression}

On observe que la diminution des résistances de tous les bétons choqués dans l'air est plus importante que celles des bétons choqués dan l'eau. Dans le premier cas les BO, BA, BAF et le BAFP ont perdu respectivement 13.5, 7, 9.1 et $10 \%$ tandis que de le deuxième cas (choc dans l'eau) ils perdent respectivement $2.5,4.5,4.1$ et $5.7 \%$. 


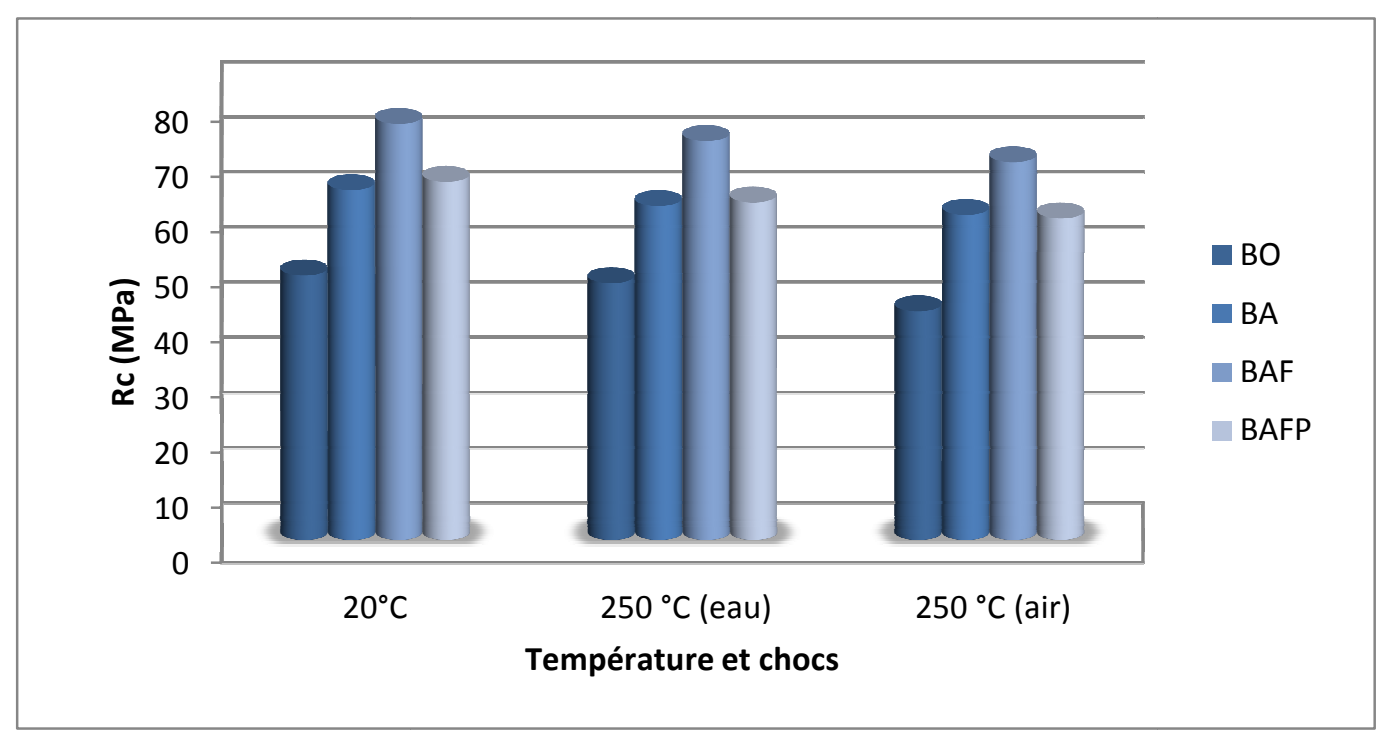

Fig.5. Effet de la température et la nature du refroidissement sur la résistance à la compression des bétons après 60 jours

La diminution du rapport E/C de 0.45 à 0.40 en gardant la même maniabilité par l'ajout de superplastifiant a augmenté la résistance de $14.4 \%$ (Rc de BA). Cet adjuvant disperse les grains de ciment par la défloculation qui aide à la libération des eaux piégées entre les grains de ciment. Ce phénomène donne à la pâte une maniabilité élevée qu'on peut diminuer par la diminution du dosage en eau et ce qui amoindrit la porosité et par conséquent augmente la résistance (figure 5).

Le BA, BAF et le BAFP peuvent être considérés comme des bétons à hautes performance (BHP) vu leurs résistances à la compression à 28 jours supérieure à $60 \mathrm{MPa}$. Le BAF atteigne 75.5 MPa, cela montre l'effet de la silice ultra fine. Le BAFP aussi contient de la silice mais sa résistance est faible de $10 \mathrm{MPa}$ (d'environs 14\%) par rapport au BAF. Cette baisse de résistance est due à l'ajout des fibres de polypropylène. Ces dernières ont une mauvaise adhésion avec la pâte liante. Ceci augmente la porosité et par conséquent diminue la résistance.

\subsection{Influence de type de choc sur la résistance à la compression}

À 60 jours, et selon la figure 6, la résistance des BA et BAFP après choc dans l'air est plus élevés que celle obtenue après choc dans l'eau. Cette observation n'a pas été enregistrée qu'au $28^{\text {ème }}$ jour. En revenant à la porosité, le BAFP a donné la forte porosité après son chauffage à $250{ }^{\circ} \mathrm{C}$, lors de son immersion dans l'eau, cette forte porosité par rapport aux autres bétons 
a permis à la pénétration de l'eau. Cette eau réagit avec la chaux et forme la portlandite, cette dernière possède un volume plus important que la $\mathrm{CaO}$. Cette augmentation du volume engendre une fissuration supplémentaire qui entraîne une réduction de la résistance en compression.

Dans le cas où le béton est choqué à l'air, l'ajout de la silice entraine au-delà de $150{ }^{\circ} \mathrm{C}$, la formation de gel de tobermorite, type de C-S-H très résistant (effet pouzzolanique).
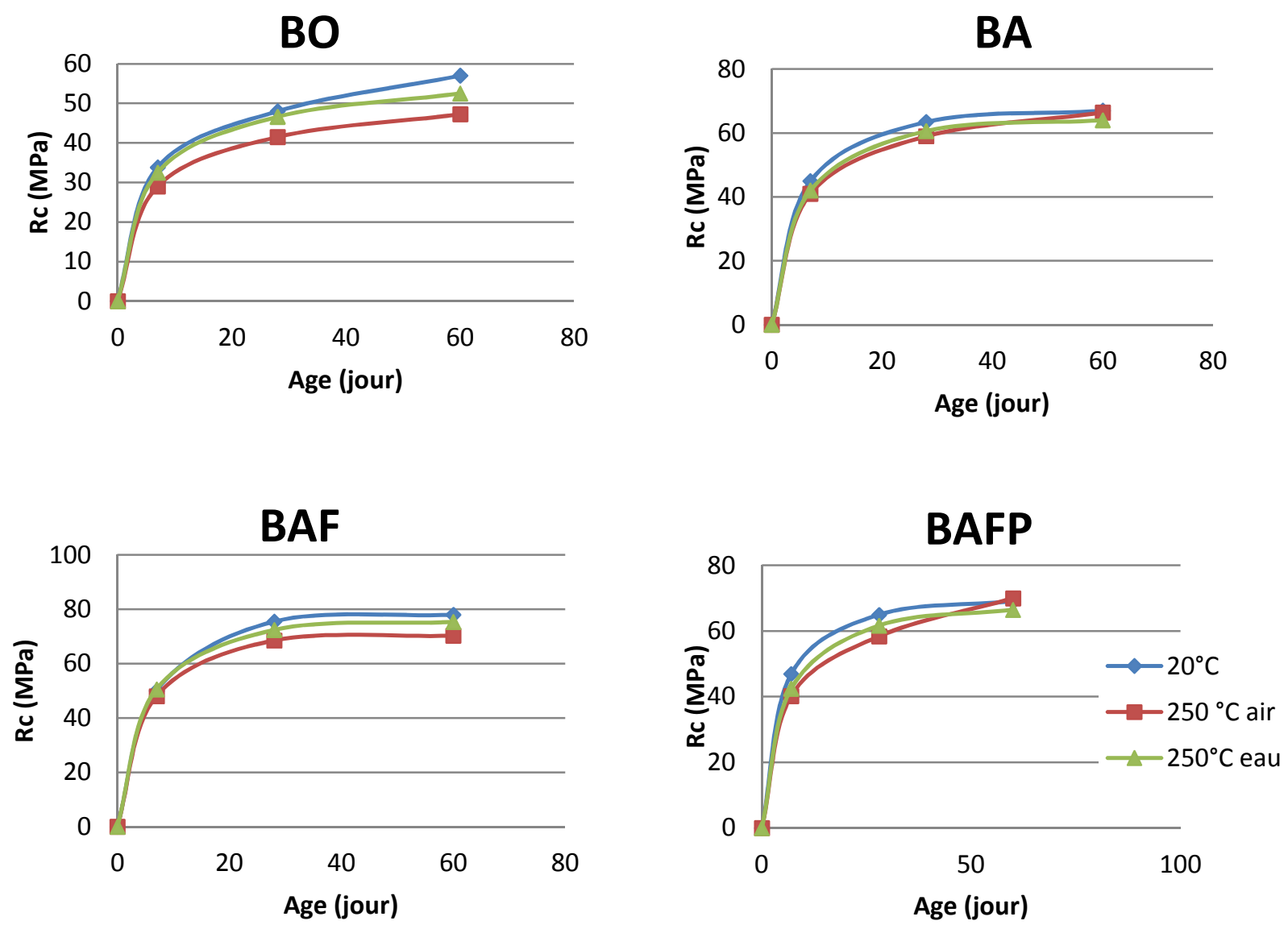

Fig.6. Résistance à la compression en fonction de l'âge pour les différents choc thermique

\section{CONCLUSION}

En utilisant les matériaux de l'ouest Algérien, qui répondants aux normes pour la fabrication d'un béton de qualité, et qui résiste aux hautes températures, notre étude a montré :

- L'effet bénéfique de l'utilisation de la silice ultra fine qui densifie la structure du béton par la diminution de la porosité (de 38\%) et l'augmentation de la résistance (de 15.8\%) à température ambiante. 
- Pour les bétons exposés au feu, en plus de la silice ultra fine, l'utilisation des fibres de polypropylène est recommandée vu la résistance élevée à long terme qui atteint celle du béton non chauffé.

- Après chauffage, le choc des bétons dans l'air à plus d'influence sur la porosité et la résistance résiduelle par rapport au choc dans l'eau.

\section{REFERENCES BIBLIOGRAPHIQUES}

[1] J.C Mindeguia, 2009; «Contribution expérimentale à la compréhension des risques d'instabilité thermique des bétons », thèse de doctorat à l'université de Pau, France.

[2] Baroghel-Bouny, Caractérisation des pâtes de ciment et des bétons, méthode, analyse, interprétation. LCPC, 1994.

[3] Noumowe «Contribution expérimentale a la compréhension des risques d'instabilité thermique des bétons », 2005, Thèse de doctorat à l'université de Pau, France.

[4] Khoury and Willoughby 2008; «Polypropylene fibres in heated concrete. Part 2: pressure relief mechanisms and modelling criteria." Magazine of Concrete Research 60(3): 189-204.

[5] Kalifa, et al 2002 «Comportement à haute température des bétons à haute performances : de l'éclatement à la microstructure ». Cahier du CSTB, n³435, Décembre 2002.

[6] I. Gaweska Hager, Comportement à haute température des bétons à haute performance évolution des principales propriétés mécaniques », 2004, Thèse de Doctorat de l'ENPC.

\section{How to cite this article:}

Benkaddour M, Senhadji Y, Kazi Aoual F and Semcha A. The Effect of High Temperatures on Concrete Incorporating Ultrafine Silica and Polypropylene Fibers. J. Fundam. Appl. Sci., 2016, 8(2), 256-267. 